J. Nonlinear Var. Anal. 2 (2018), No. 1, pp. 49-61

Available online at http://jnva.biemdas.com

https://doi.org/10.23952/jnva.2.2018.1.05

\title{
TWO SIMPLE RELAXED PERTURBED EXTRAGRADIENT METHODS FOR SOLVING VARIATIONAL INEQUALITIES IN EUCLIDEAN SPACES
}

\author{
AVIV GIBALI \\ Department of Mathematics, ORT Braude College, 2161002 Karmiel, Israel
}

\begin{abstract}
The Korpelevich's extragradient method is an iterative method designed for solving the variational inequality problem (VIP) and also can be used for other problems, such as finding saddle-points. The method employs two orthogonal projections onto the feasible set of the VIP per each iteration. This method was studied intensively and many generalizations and extensions were proposed along the years. Censor et al. proposed some modifications of the method in Euclidean as well as in Hilbert spaces, including a perturbed version which allows projections onto the members of an infinite sequence of subsets that epi-converges to the feasible set of the VIP. In this paper study this extragradient variant and extend it further to two relaxed and perturbed algorithms by using the properties of the involved operators and the perturbed sets.

Keywords. Epi-convergence; Extragradient method; Relaxed perturbed extragradient method; Lipschitz mapping; Variational inequality.
\end{abstract}

2010 Mathematics Subject Classification. 47H09, 47H14, 47J20, 65K15.

\section{INTRODUCTION}

We are concerned here with the Variational Inequality Problem (VIP) in Euclidean spaces, which consists in finding a point $x^{*}$ such that

$$
x^{*} \in C \text { and }\left\langle\mathscr{F}\left(x^{*}\right), x-x^{*}\right\rangle \geq 0, \quad \forall x \in C,
$$

where $\mathscr{F}: \mathbb{R}^{n} \rightarrow \mathbb{R}$ is a given mapping, $C$ is a non-empty, closed and convex subset of $\mathbb{R}$ and $\langle\cdot, \cdot\rangle$ denotes the inner product in $\mathbb{R}^{n}$. We denote the solution set of $(1.1)$ by $\operatorname{Sol}(\mathscr{F}, C)$. Korpelevich [15] proposed the Extragradient Method for solving the VIP; see also Antipin [1] and the excellent and intensive books of Facchinei and Pang [12, Chapter 12]. In each iteration of Korpelevich's algorithm, in order to get the next iterate $x^{k+1}$, two orthogonal projections onto $C$ are calculated, according to the following iterative step. Given the current iterate $x^{k}$, calculate

$$
\begin{gathered}
y^{k}=P_{C}\left(x^{k}-\tau \mathscr{F}\left(x^{k}\right)\right), \\
x^{k+1}=P_{C}\left(x^{k}-\tau \mathscr{F}\left(y^{k}\right)\right),
\end{gathered}
$$

where $\tau$ is some positive number and $P_{C}$ denotes the Euclidean nearest-point projection onto $C$.

Censor, Gibali and Reich in [10] proposed two extensions of Korpelevich's extragradient method. The second extension, called the perturbed extragradient algorithm, allows projections onto the members of an infinite sequence of subsets $\left\{C_{k}\right\}_{k=0}^{\infty}$ of $C$ which epi-converges to $C$ (the feasible set of the VIP

E-mail address: avivg@braude.ac.il.

Received July 10, 2017; Accepted August 25, 2017.

(c)2018 Journal of Nonlinear and Variational Analysis 
(1.1)). In this paper, we show how by including standard assumptions on $\mathscr{F}$, for example, $C \cap \operatorname{Zer}(\mathscr{F}) \neq$ $\emptyset$, where $\operatorname{Zer}(\mathscr{F}):=\left\{x \in \mathbb{R}^{n} \mid \mathscr{F}(x)=0\right\}$, the algorithm convergence to a zero of the mapping $\mathscr{F}$. Using a generalization of the Krasnosel'skiǔ-Mann iterative step, as proposed in [23], we obtain a proof of convergence of a relaxed version of the perturbed extragradient method. For an excellent book in this area, see Zaslavski [22]. In particular, Chapters 12 and 13 are focus on solving variational inequalities by the extragradient method with perturbations.

The paper is organized as follows. In Section 2 we list several known facts about functions and mappings that we need in the sequel. In Section 3 and 4 two relaxed perturbed extragradient algorithms are presented and analyzed.

\section{PRELIMINARIES}

Before we recall the perturbed extragradient algorithm [10, Algorithm 4.3] we present several definitions and notations. Following Santos and Scheimberg [21] we denote by $\operatorname{NCCS}\left(\mathbb{R}^{n}\right)$ the family of all non-empty, closed and convex subsets of $\mathbb{R}^{n}$.

Let $C$ be non-empty, closed and convex subset of $\mathbb{R}^{n}$, that is $C \in \operatorname{NCCS}\left(\mathbb{R}^{n}\right)$. For each point $x \in \mathbb{R}^{n}$, there exists a point $P_{C}(x)$ in $C$ that is the unique point in $C$ closest to $x$, in the sense of the Euclidean norm; that is,

$$
\left\|x-P_{C}(x)\right\| \leq\|x-y\|, \quad \forall y \in C .
$$

The mapping $P_{C}: \mathbb{R}^{n} \rightarrow C$ is called the orthogonal or metric projection of $\mathbb{R}^{n}$ onto $C$. It is well known that $P_{C}$ is a non-expansive mapping on $\mathbb{R}^{n}$, i.e.,

$$
\left\|P_{C}(x)-P_{C}(y)\right\| \leq\|x-y\|, \quad \forall x, y \in \mathbb{R}^{n} .
$$

The metric projection $P_{C}$ is characterized [13, Section 3] by the following two properties:

$$
P_{C}(x) \in C
$$

and

$$
\left\langle x-P_{C}(x), P_{C}(x)-y\right\rangle \geq 0, \quad \forall x \in \mathbb{R}^{n}, y \in C,
$$

and if $C$ is a hyperplane, then (2.4) becomes an equality. Another useful property of the metric projection is

$$
\|x-y\|^{2} \geq\left\|x-P_{C}(x)\right\|^{2}+\left\|y-P_{C}(x)\right\|^{2}, \quad \forall x \in \mathbb{R}^{n}, y \in C .
$$

Definition 2.1. Let $\mathscr{F}: \mathbb{R}^{n} \rightarrow \mathbb{R}^{n}$ be a given mapping. The fixed point set of $\mathscr{F}$ is defined as

$$
\operatorname{Fix}(\mathscr{F}):=\left\{x \in \mathbb{R}^{n} \mid \mathscr{F}(x)=x\right\}
$$

and the zero set of $\mathscr{F}$ is

$$
\operatorname{Zer}(\mathscr{F}):=\left\{x \in \mathbb{R}^{n} \mid \mathscr{F}(x)=0\right\} .
$$

A well-known relation between the solution set of the VIP (1.1), $\operatorname{Sol}(\mathscr{F}, C)$, and the fixed point set of the operator $P_{C}(I-\lambda \mathscr{F})$ is: for any $\lambda \geq 0$;

$$
\operatorname{Sol}(\mathscr{F}, C)=\operatorname{Fix}\left(P_{C}(I-\lambda \mathscr{F})\right),
$$

see e.g., Eaves [11].

Next we present two useful results which will be needed for our convergence theorem (see, e.g., [12, Proposition 1.5.9 and Exercise 1.8.29]). 
Lemma 2.1. Let $C \subset \mathbb{R}^{n}$ be non-empty, closed and convex. Let $\mathscr{F}: C \rightarrow \mathbb{R}^{n}$. A point $x$ belongs to $\operatorname{Sol}(\mathscr{F}, C)$ if and only if there exists a point $z$ such that $x=P_{C}(z)$ and $\mathscr{F}\left(P_{C}(z)\right)+z-P_{C}(z)=0$.

The mapping $\mathscr{F} \circ P_{C}+I-P_{C}$ is known as the normal operator, for more details see [5, Chapter 8] and [12, Chapter 1].

Definition 2.2. Let $\mathscr{F}: \mathbb{R}^{n} \rightarrow \mathbb{R}^{n}$ be a given mapping and $C \subseteq \mathbb{R}^{n}$.

(i) The mapping $\mathscr{F}$ is called Lipschitz continuous on $C$ with constant $L>0$ if

$$
\|\mathscr{F}(x)-\mathscr{F}(y)\| \leq L\|x-y\|, \quad \forall x, y \in C .
$$

(ii) The mapping $\mathscr{F}$ is called monotone on $C$ if

$$
\langle\mathscr{F}(x)-\mathscr{F}(y), x-y\rangle \geq 0, \quad \forall x, y \in C .
$$

(iii) $\mathscr{F}$ is called $\alpha$-inverse strongly monotone ( $\alpha$-ISM) on $C$ if

$$
\langle\mathscr{F}(x)-\mathscr{F}(y), x-y\rangle \geq \alpha\|\mathscr{F}(x)-\mathscr{F}(y)\|^{2}, \quad \forall x, y \in C
$$

this property is also known as the Dunn property or cocoercivity.

(iv) $\mathscr{F}$ is called firmly nonexpansive on $C$ if

$$
\langle\mathscr{F}(x)-\mathscr{F}(y), x-y\rangle \geq\|\mathscr{F}(x)-\mathscr{F}(y)\|^{2}, \quad \forall x, y \in C,
$$

i.e., if it is 1-ISM.

(v) The mapping $\mathscr{F}$ is called pseudo-monotone if

$$
\langle\mathscr{F}(y), x-y\rangle \geq 0 \Rightarrow\langle\mathscr{F}(x), x-y\rangle \geq 0 .
$$

(vi) $\mathscr{F}$ is called averaged [4] if there exists a non-expansive operator $N$ and a number $c \in(0,1)$ such that

$$
\mathscr{F}=(1-c) I+c N
$$

where $I$ is the identity operator. In this case we also say that $\mathscr{F}$ is $c$-av [6].

Remark 2.1. (i) It can be verified that if $\mathscr{F}$ is $\alpha$-ISM, then it is Lipschitz continuous with constant $L=1 / \alpha$.

(ii) It is known that an operator $\mathscr{F}$ is averaged if and only if its complement $I-\mathscr{F}$ is $\alpha$-ISM for some $\alpha>1 / 2$; see, e.g., [6, Lemma 2.1].

(iii) The operator $\mathscr{F}$ is firmly nonexpansive if and only if its complement $I-\mathscr{F}$ is firmly nonexpansive. The operator $h$ is firmly nonexpansive if and only if $h$ is $(1 / 2)$-av (see [13, Proposition 11.2] and [6, Lemma 2.3]).

(iv) If $\mathscr{F}_{1}$ and $\mathscr{F}_{2}$ are $c_{1}$-av and $c_{2}$-av, respectively, then their composition $S=\mathscr{F}_{1} \mathscr{F}_{2}$ is $\left(c_{1}+c_{2}-\right.$ $\left.c_{1} c_{2}\right)$-av. See [6, Lemma 2.2].

Lemma 2.2. Let $C \subseteq \mathbb{R}^{n}$ be non-empty, closed and convex. Let $\mathscr{F}: C \rightarrow \mathbb{R}^{n}$ be Lipschitz continuous with constant $L>0$. For any $\lambda \in(0,1 / L)$, we get

$$
\operatorname{Sol}(\mathscr{F}, C)=\operatorname{Fix}\left(P_{C}\left(I-\lambda \mathscr{F}\left(P_{C}(I-\lambda \mathscr{F})\right)\right)\right) .
$$


Proof. (i) Let $x \in \operatorname{Sol}(\mathscr{F}, C)$. Applying (2.8) twice, we get

$$
P_{C}\left(I-\lambda \mathscr{F}\left(P_{C}(x-\lambda \mathscr{F}(x))\right)\right)=P_{C}(I-\lambda \mathscr{F}(x))=x,
$$

which implies that $x \in \operatorname{Fix}\left(P_{C}\left(I-\lambda \mathscr{F}\left(P_{C}(I-\lambda \mathscr{F})\right)\right)\right)$.

(ii) On the other hand, let $x \in F i x\left(P_{C}\left(I-\lambda \mathscr{F}\left(P_{C}(I-\lambda \mathscr{F})\right)\right)\right.$. Denote by $y:=P_{C}(x-\lambda \mathscr{F}(x))$, we get $x=P_{C}(x-\lambda F(y))$. We now show that $x=y$. Indeed, following the non-expansiveness of the metric projection $P_{C}$ and the Lipschitz continuity of $\mathscr{F}$

$$
\begin{aligned}
\|x-y\| & =\left\|P_{C}(x-\lambda \mathscr{F}(y))-P_{C}(x-\lambda \mathscr{F}(x))\right\| \\
& \leq\|(x-\lambda \mathscr{F}(y))-(x-\lambda \mathscr{F}(x))\|=\lambda\|\mathscr{F}(x)-\mathscr{F}(y)\| \\
& \leq \frac{\lambda}{L}\|x-y\| .
\end{aligned}
$$

Following the assumption on $\lambda$, we get that $x=y$, meaning that $x=y=P_{C}(x-\lambda \mathscr{F}(x))$, i.e., $x \in$ $\operatorname{Sol}(\mathscr{F}, C)$.

By converting this relation into an iterative method for solving the VIP (1.1), we get Korpelevich's extragradient method ((1.2)-(1.3)).

The next lemma shows when the only solution of a VIP (1.1) is a zero of the involved mapping $\mathscr{F}$.

Lemma 2.3. Let $C \subseteq \mathbb{R}^{n}$ be nonempty, closed and convex set and $\mathscr{F}: \mathbb{R}^{n} \rightarrow \mathbb{R}^{n}$ be $\alpha$-ISM mapping. Assume that $C \cap \operatorname{Zer}(\mathscr{F}) \neq \emptyset$, then $\operatorname{Sol}(\mathscr{F}, C)=\operatorname{Zer}(\mathscr{F})$.

Proof. First assume that $x^{*} \in \operatorname{Zer}(\mathscr{F})$, then $x^{*} \in \operatorname{Sol}(\mathscr{F}, C)$ as (1.1) holds trivially. On the other hand, assume that $x^{*} \in \operatorname{Sol}(\mathscr{F}, C)$, then by (2.8), i.e., $x^{*}=P_{C}\left(x^{*}-\lambda \mathscr{F}\left(x^{*}\right)\right)$. It can be easily proved that the mapping $I-\lambda \mathscr{F}$ is nonexpansive for $[0,2 \alpha]$ (averaged for $\lambda \in(0,2 \alpha))$ and then for $z \in C \cap F i x(I-$ $\lambda \mathscr{F})=C \cap \operatorname{Zer}(\mathscr{F})$ we have

$$
\left\|(I-\lambda \mathscr{F})\left(x^{*}\right)-z\right\|^{2} \leq\left\|x^{*}-z\right\|^{2} .
$$

Following (2.5) with $(I-\lambda \mathscr{F})\left(x^{*}\right)$ as $x$ there and $z$ as $y$ there, we get

$$
\begin{aligned}
\left\|(I-\lambda \mathscr{F})\left(x^{*}\right)-z\right\|^{2} & \geq \\
& \left\|(I-\lambda \mathscr{F})\left(x^{*}\right)-P_{C}\left(x^{*}-\lambda \mathscr{F}\left(x^{*}\right)\right)\right\|^{2} \\
& +\left\|z-P_{C}\left(x^{*}-\lambda \mathscr{F}\left(x^{*}\right)\right)\right\|^{2} .
\end{aligned}
$$

Using (2.8), we get

$$
\begin{aligned}
\left\|(I-\lambda \mathscr{F})\left(x^{*}\right)-z\right\|^{2} & \geq \\
& \left\|(I-\lambda \mathscr{F})\left(x^{*}\right)-x^{*}\right\|^{2}+\left\|z-x^{*}\right\|^{2} .
\end{aligned}
$$

The above inequality with the nonexpansiveness of $I-\lambda \mathscr{F}$ yields

$$
\left\|z-x^{*}\right\|^{2}+\left\|(I-\lambda \mathscr{F})\left(x^{*}\right)-x^{*}\right\|^{2} \leq\left\|x^{*}-z\right\|^{2} .
$$

Hence, $\left\|(I-\lambda \mathscr{F})\left(x^{*}\right)-x^{*}\right\|^{2}=0$. Since $\lambda>0$, we get that $\mathscr{F}\left(x^{*}\right)=0$, meaning that $x^{*} \in \operatorname{Zer}(\mathscr{F})$ and the proof is complete. 
Definition 2.3. [2, Proposition 3.21] Let $C$ and $\left\{C_{k}\right\}_{k=0}^{\infty}$ be a set and a sequence of sets in NCCS $\left(\mathbb{R}^{n}\right)$, respectively. The sequence $\left\{C_{k}\right\}_{k=0}^{\infty}$ is said to epi-converge to the set $C$ (denoted by $C_{k} \stackrel{\text { epi }}{\rightarrow} C$ ) if the following two conditions hold:

(i) for every $x \in C$, there exists a sequence $\left\{x^{k}\right\}_{k=0}^{\infty}$ such that $x^{k} \in C_{k}$ for all $k \geq 0$, and $\lim _{k \rightarrow \infty} x^{k}=x$;

(ii) if $x^{k_{j}} \in C_{k_{j}}$ for all $j \geq 0$, and $\lim _{j \rightarrow \infty} x^{k_{j}}=x$, then $x \in C$.

Definition 2.4. [3] Let $T$ and $U$ be non-expansive mappings. The $\gamma$-distance between $T$ and $U$ is defined as

$$
D_{\gamma}(U, T):=\sup \{\|U(x)-T(x)\| \mid\|x\| \leq \gamma\} .
$$

A related definition for distance between sets is given next.

Definition 2.5. Let $C_{1}$ and $C_{2}$ be in $\operatorname{NCCS}\left(\mathbb{R}^{n}\right)$ and $\gamma \geq 0$. The $\gamma$-distance between $C_{1}$ and $C_{2}$ is defined as

$$
\begin{aligned}
d_{\gamma}\left(C_{1}, C_{2}\right) & :=\sup \left\{\left\|P_{C_{1}}(x)-P_{C_{2}}(x)\right\| \mid\|x\| \leq \gamma\right\} \\
& =D_{\gamma}\left(P_{C_{1}}, P_{C_{2}}\right) .
\end{aligned}
$$

Another close and related definition is the following.

Definition 2.6. Let $C_{1}$ and $C_{2}$ be in $\operatorname{NCCS}\left(\mathbb{R}^{n}\right)$. The Hausdorff metric is defined by

$$
d_{H}\left(C_{1}, C_{2}\right):=\max \left\{\sup _{x \in C_{2}} d\left(x, C_{1}\right), \sup _{y \in C_{1}} d\left(y, C_{2}\right)\right\},
$$

where the distance function is defined by $d(x, C):=\inf \{\|x-z\| \mid z \in C\}$.

The next proposition is [21, Proposition 7], but its Banach space variant already appears in [14, Proposition 7]. For completeness, we also include the proof of the proposition.

Proposition 2.1. Let $C$ and $\left\{C_{k}\right\}_{k=0}^{\infty}$ be a set and a sequence of sets in $\operatorname{NCCS}\left(\mathbb{R}^{n}\right)$, respectively. If $C_{k} \stackrel{\text { epi }}{\rightarrow} C$ and $\lim _{k \rightarrow \infty} y^{k}=y$, then

$$
\lim _{k \rightarrow \infty} P_{C_{k}}\left(y^{k}\right)=P_{C}(y)
$$

Consequently, we also have

$$
\lim _{k \rightarrow \infty} P_{C_{k}}(y)=P_{C}(y)
$$

for all $y$.

Proof. Let $x=P_{C}(y)$. Since $x \in C$, there is a sequence $\left\{x^{k}\right\}_{k=0}^{\infty}$ converging to $x$, with $x^{k} \in C_{k}$, for each $k$. Using

$$
\left\|P_{C_{k}}(x)-x\right\| \leq\left\|x^{k}-x\right\|
$$

we conclude that the sequence $\left\{P_{C_{k}}(x)\right\}_{k=0}^{\infty}$ also converges to $x$. From

$$
\left\|P_{C_{k}}(y)-P_{C_{k}}(x)\right\| \leq\|y-x\|,
$$

it follows that the sequence $\left\{P_{C_{k}}(y)\right\}_{k=0}^{\infty}$ is bounded. From

$$
\left\|P_{C_{k}}\left(y^{k}\right)-P_{C_{k}}(y)\right\| \leq\left\|y^{k}-y\right\|,
$$


it follows that the sequence $\left\{P_{C_{k}}\left(y^{k}\right)\right\}_{k=0}^{\infty}$ is bounded. Then there is a $z \in C$ and a subsequence $\left\{P_{C_{k_{n}}}\left(y^{k_{n}}\right)\right\}_{n=0}^{\infty}$ converging to $z$. Because

$$
\left\|P_{C_{k_{n}}}\left(y^{k_{n}}\right)-y^{k_{n}}\right\| \leq\left\|P_{C_{k_{n}}}(x)-y^{k_{n}}\right\|,
$$

taking limits, we conclude that

$$
\|z-y\| \leq\|x-y\|
$$

Therefore, $z=P_{C}(y)=x$.

A useful result is the following.

Theorem 2.1. Let $T$ and $\left\{T_{k}\right\}_{k=0}^{\infty}$ be non-expansive mappings. Then the following assertions are equivalent.

(i) $\lim _{k \rightarrow \infty} T_{k}(x)=T(x)$ for all $x$;

(ii) $\lim _{k \rightarrow \infty}\left\|T_{k}(x)-T(x)\right\|=0$; uniformly on bounded sets;

(iii) $\lim _{k \rightarrow \infty} D_{\gamma}\left(T_{k}, T\right)=0$ for all $\gamma \geq 0$.

Proof. We show that (i) implies (ii). Other implications in the theorem are obvious. If (ii) does not hold, then we can find $\gamma>0$ and $\varepsilon>0$ such that, for every positive integer $n$, there is $k_{n} \geq n$ and $x^{k_{n}}$, with $\left\|x^{k_{n}}\right\| \leq \gamma$, and

$$
\left\|T_{k_{n}}\left(x^{k_{n}}\right)-T\left(x^{k_{n}}\right)\right\| \geq \varepsilon
$$

Again, without loss of generality, and to simplify notation, we assume that the sequence $\left\{x^{k_{n}}\right\}_{n=0}^{\infty} \rightarrow x^{*}$. We have

$$
\begin{aligned}
\left\|T_{k_{n}}\left(x^{k_{n}}\right)-T\left(x^{k_{n}}\right)\right\| & \leq\left\|T_{k_{n}}\left(x^{k_{n}}\right)-T_{k_{n}}\left(x^{*}\right)\right\|+\left\|T_{k_{n}}\left(x^{*}\right)-T\left(x^{*}\right)\right\| \\
& +\left\|T\left(x^{*}\right)-T\left(x^{k_{n}}\right)\right\| \\
& \leq\left\|x^{k_{n}}-x^{*}\right\|+\left\|T_{k_{n}}\left(x^{*}\right)-T\left(x^{*}\right)\right\|+\left\|x^{*}-x^{k_{n}}\right\| .
\end{aligned}
$$

All three terms in the last line converge to zero, as $n \rightarrow \infty$. Consequently, (ii) must hold.

As a special case of the above theorem, Proposition 2.1 can be strengthened as follows; see also [3, Corollary 2.53] and [21, Lemma 6].

Theorem 2.2. Let $C$ and $\left\{C_{k}\right\}_{k=0}^{\infty}$ be a set and a sequence of sets in $\operatorname{NCCS}\left(\mathbb{R}^{n}\right)$, respectively. Then the following assertions are equivalent.

(i) $\lim _{k \rightarrow \infty} P_{C_{k}}(z)=P_{C}(z)$ for all $z$;

(ii) $C_{k} \stackrel{\text { epi }}{\rightarrow} C$;

(iii) $\lim _{k \rightarrow \infty}\left\|P_{C_{k}}(z)-P_{C}(z)\right\|=0$; uniformly on bounded sets;

(iv) $\lim _{k \rightarrow \infty} d_{\gamma}\left(C_{k}, C\right)=0$ for all $\gamma \geq 0$.

Proof. We show that (i) implies (ii) and (i) implies (iii). Other implications in the theorem are obvious. Assume that (i) holds. Let $x \in C$. Then $x^{k}=P_{C_{k}}(x) \in C_{k}$ and $x^{k} \rightarrow x$. Now let $y^{k_{n}} \rightarrow y$, and $y^{k_{n}} \in C_{k_{n}}$. We have

$$
\begin{aligned}
\left\|y^{k_{n}}-P_{C}(y)\right\| & \leq\left\|y^{k_{n}}-P_{C_{k_{n}}}(y)\right\|+\left\|P_{C_{k_{n}}}(y)-P_{C}(y)\right\| \\
& \leq\left\|y^{k_{n}}-y\right\|+\left\|P_{C_{k_{n}}}(y)-P_{C}(y)\right\|
\end{aligned}
$$


and both terms in this sum converge to zero as $n \rightarrow \infty$. So $C_{k} \stackrel{\text { epi }}{\rightarrow} C$.

Now we show that (i) implies (iii). If (iii) does not hold, then there are $\gamma>0$ and $\varepsilon>0$ such that, for every positive integer $n$, there is $k_{n} \geq n$ and $x^{k_{n}} \in C_{k_{n}}$, with $\left\|x^{k_{n}}\right\| \leq \gamma$, and

$$
\left\|P_{C_{k_{n}}}\left(x^{k_{n}}\right)-P_{C}\left(x^{k_{n}}\right)\right\| \geq \varepsilon \text {. }
$$

Without loss of generality, and to simplify notation, we assume that the sequence $\left\{x^{k_{n}}\right\}_{n=0}^{\infty} \rightarrow x^{*}$. We then have

$$
\begin{aligned}
\left\|P_{C_{k_{n}}}\left(x^{k_{n}}\right)-P_{C}\left(x^{k_{n}}\right)\right\| & \leq\left\|P_{C_{k_{n}}}\left(x^{k_{n}}\right)-P_{C_{k_{n}}}\left(x^{*}\right)\right\|+\left\|P_{C_{k_{n}}}\left(x^{*}\right)-P_{C}\left(x^{*}\right)\right\| \\
& +\left\|P_{C}\left(x^{*}\right)-P_{C}\left(x^{k_{n}}\right)\right\| \\
& \leq\left\|x^{k_{n}}-x^{*}\right\|+\left\|P_{C_{k_{n}}}\left(x^{*}\right)-P_{C}\left(x^{*}\right)\right\|+\left\|x^{*}-x^{k_{n}}\right\| .
\end{aligned}
$$

All three of the terms in the last line converge to zero as $n \rightarrow \infty$. Therefore, (iii) must hold. This completes the proof.

Next we present the well-known Krasnosel'skiü-Mann-Opial Theorem [16, 17, 20]; as a matter of fact, Opial's Theorem is more general than the following.

Theorem 2.3. [16, 17, 20] Let $\mathscr{H}$ be a real Hilbert space and $C \subset \mathscr{H}$ be a non-empty, closed and convex subset of $\mathscr{H}$. Given an averaged operator $h: C \rightarrow C$ with $F i x(h) \neq \emptyset$ and an arbitrary $x^{0} \in C$, the sequence generated by the recursion $x^{k+1}=h\left(x^{k}\right), k \geq 0$, converges weakly to a point $z \in F i x(h)$.

Several generalizations of this theorem were presented and studied where $h$ is replaced by a sequence of mappings $\left\{h_{k}\right\}_{k=0}^{\infty}$, see e.g., [7, 8, 23] and [9]. We will focus here on the generalization proposed by Yang and Zhao [23], although others can be applied as well.

Theorem 2.4. [23, Theorem 2.3] Let $N$ and $\left\{N_{k}\right\}_{k=0}^{\infty}$ be non-expansive mappings on a Hilbert space $\mathscr{H}$, for $k=0,1, \ldots, N_{k} \rightarrow N$ and $\alpha_{k} \in(0,1)$ satisfy $\sum_{k=0}^{\infty} \alpha_{k}\left(1-\alpha_{k}\right)=+\infty$. Then the sequence $\left\{x^{k}\right\}_{k=0}^{\infty}$ defined by the iterative step

$$
x^{k+1}=\left(1-\alpha_{k}\right) x^{k}+\alpha_{k} N_{k}\left(x^{k}\right)
$$

converges weakly to a fixed point of $N$, provided $\sum_{k=0}^{\infty} \alpha_{k} D_{\gamma}\left(N_{k}, N\right)<+\infty$ for any given $\gamma>0$, whenever such fixed points exist.

\section{THE PERTURBED EXTRAGRADIENT ALGORITHM}

Now we recall the perturbed extragradient algorithm.

\section{Algorithm 3.1. The perturbed extragradient algorithm}

Step 0: Let $\left\{C_{k}\right\}_{k=0}^{\infty}$ be a sequence of sets in $\operatorname{NCCS}\left(\mathbb{R}^{n}\right)$ such that $C_{k} \stackrel{\text { epi }}{\rightarrow} C$. Select a starting point $x^{1} \in C_{0}$ and $\tau>0$, and set $k=1$.

Step 1: Given the current iterate $x^{k} \in C_{k-1}$, compute

$$
y^{k}=P_{C_{k}}\left(x^{k}-\tau \mathscr{F}\left(x^{k}\right)\right)
$$

and

$$
x^{k+1}=P_{C_{k}}\left(x^{k}-\tau \mathscr{F}\left(y^{k}\right)\right) .
$$

Step 2: Set $k \leftarrow(k+1)$ and return to Step 1. 


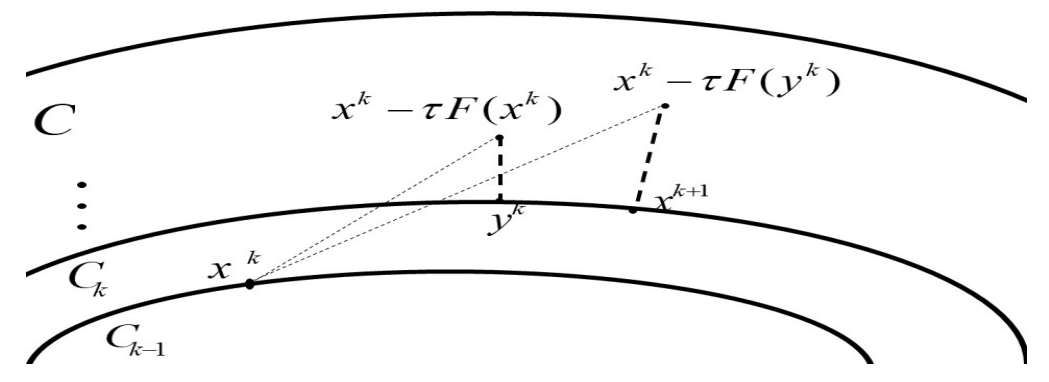

FIGURE 1. In the iterative step of Algorithm 3.1, $x^{k+1}$ is obtained by performing the projections of the original Korpelevich method with respect to the set $C_{k}$.

In order to prove the convergence of Algorithm 3.1 we make the following assumptions (also used in [10]).

Condition 3.1. The solution set of $(1.1)$, that is $\operatorname{Sol}(\mathscr{F}, C) \neq \emptyset$.

Condition 3.2. The mapping $\mathscr{F}$ is $\alpha$-ISM on $C$.

Condition 3.3. The mapping $\mathscr{F}\left(P_{C}(I-\tau \mathscr{F})\right)$ is ISM on $C$.

The convergence theorem [10, Theorem 5.2] is next.

Theorem 3.1. Assume that $C_{k} \subseteq C_{k+1} \subseteq$ C for all $k \geq 0$, that $C_{k} \stackrel{e p i}{\rightarrow} C$, and that Conditions 3.1-3.3 hold. Let $\tau<1 / L(=\alpha)$. Then any sequence $\left\{x^{k}\right\}_{k=0}^{\infty}$, generated by Algorithm 3.1, converges to a solution of (1.1).

Now, following [21] we present the relaxed version of the perturbed extragradient algorithm where instead of pseudo monotonicity we assume inverse strong monotonicity with respect to one solution $x^{*} \in$ $\operatorname{Sol}(\mathscr{F}, C)$. In what follows, we use the following notation.

$$
\begin{aligned}
& T(x):=P_{C}\left(x-\tau \mathscr{F}\left(P_{C}(x-\tau \mathscr{F}(x))\right)\right), \\
& T_{k}(x):=P_{C_{k}}\left(x-\tau \mathscr{F}\left(P_{C_{k}}(x-\tau \mathscr{F}(x))\right)\right) .
\end{aligned}
$$

Observe that the iterative step of Algorithm 3.1 can be written as

$$
x^{k+1}=T_{k}\left(x^{k}\right) .
$$

Let $\left\{C_{k}\right\}_{k=0}^{\infty}$ be a sequence of sets in $\operatorname{NCCS}\left(\mathbb{R}^{n}\right)$ such that $C_{k} \stackrel{\text { epi }}{\rightarrow} C$. For the relaxed perturbed extragradient algorithm we take $T$ and $T_{k}$ as in (3.3), and $\alpha_{k} \in(0,1)$ satisfying $\sum_{k=0}^{\infty} \alpha_{k}\left(1-\alpha_{k}\right)=+\infty$.

\section{Algorithm 3.2. The relaxed perturbed extragradient algorithm}

Step 0: Select a starting point $x^{1} \in C_{0}$ and $\tau>0$, and set $k=1$.

Step 1: Given the current iterate $x^{k} \in C_{k-1}$, compute the next iterate as

$$
x^{k+1}=\left(1-\alpha_{k}\right) x^{k}+\alpha_{k} T_{k}\left(x^{k}\right)
$$

Step 2: Set $k \leftarrow(k+1)$ and return to Step 1 . 
3.1. Convergence of the relaxed perturbed extragradient algorithm. For proving the convergence of Algorithm 3.2, we apply Theorem 2.4. In order to do that we first need to show that $T$ and $\left\{T_{k}\right\}_{k=0}^{\infty}$ are non-expansive mappings. This result is similar to [21, Proposition 11].

Proposition 3.1. Assume that $C_{k} \subseteq C_{k+1} \subseteq C$ for all $k \geq 0$. In addition, assume that Conditions 3.2 and 3.3 hold $(\alpha$-ISM on $C)$ and choose $\tau \in[0,2 \alpha]$. Then both $T$ and $T_{k}$ are non-expansive on $C$; that is

$$
\left\|T_{k}(x)-T_{k}(y)\right\| \leq\|x-y\|, \quad \forall x, y \in C
$$

and

$$
\|T(x)-T(y)\| \leq\|x-y\|, \quad \forall x, y \in C .
$$

Proof. Let $x, y \in C$. By the definition of $T_{k}$ (see (3.3)), the non-expansiveness of the metric projection $P_{C_{k}}$ and the inclusion $C_{k} \subseteq C_{k+1} \subseteq C$, we get

$$
\begin{aligned}
\left\|T_{k}(x)-T_{k}(y)\right\|^{2} & =\left\|P_{C_{k}}\left(x-\tau \mathscr{F}\left(P_{C_{k}}(x-\tau \mathscr{F}(x))\right)\right)-P_{C_{k}}\left(y-\tau \mathscr{F}\left(P_{C_{k}}(y-\tau \mathscr{F}(y))\right)\right)\right\|^{2} \\
& \leq\left\|\left(x-\tau \mathscr{F}\left(P_{C_{k}}(x-\tau \mathscr{F}(x))\right)\right)-\left(y-\tau \mathscr{F}\left(P_{C_{k}}(y-\tau \mathscr{F}(y))\right)\right)\right\|^{2} \\
& =\left\|(x-y)-\tau\left(\mathscr{F}\left(P_{C_{k}}(x-\tau \mathscr{F}(x))\right)-\mathscr{F}\left(P_{C_{k}}(y-\tau \mathscr{F}(y))\right)\right)\right\|^{2} \\
& =\|x-y\|^{2}-2 \tau\left\langle x-y, \mathscr{F}\left(P_{C_{k}}(x-\tau \mathscr{F}(x))\right)-\mathscr{F}\left(P_{C_{k}}(y-\tau \mathscr{F}(y))\right)\right\rangle \\
& +\tau^{2}\left\|\mathscr{F}\left(P_{C_{k}}(x-\tau \mathscr{F}(x))\right)-\mathscr{F}\left(P_{C_{k}}(y-\tau \mathscr{F}(y))\right)\right\|^{2} \\
& \leq\|x-y\|^{2}+\left(\tau^{2}-2 \tau \alpha\right)\left\|\mathscr{F}\left(P_{C_{k}}(x-\mathscr{F}(x))\right)-\mathscr{F}\left(P_{C_{k}}(y-\mathscr{F}(y))\right)\right\|^{2} .
\end{aligned}
$$

With $\tau \in[0,2 \alpha]$ we get that $T_{k}$ is non-expansive on $C$. Following similar arguments, the non-expansivity of $T$ is obtained.

Proposition 3.2. Assume that $C_{k} \subseteq C_{k+1} \subseteq C$ such that $C_{k} \stackrel{e p i}{\rightarrow} C$. In addition, assume that Conditions 3.2 and 3.3 hold ( $\alpha$-ISM on $C)$ and choose $\tau \in[0,2 \alpha]$. Then $T_{k} \rightarrow T$.

Proof. Let $x \in \mathbb{R}^{n}$ and let $\|x\| \leq \gamma$ for $\gamma \geq 0$. Then, following the non-expansiveness of the metric projections $P_{C}$ and $P_{C_{k}}$ we obtain.

$$
\begin{aligned}
\left\|T_{k}(x)-T(x)\right\| & =\left\|P_{C_{k}}\left(x-\tau \mathscr{F}\left(P_{C_{k}}(x-\tau \mathscr{F}(x))\right)\right)-P_{C}\left(x-\tau \mathscr{F}\left(P_{C}(x-\tau \mathscr{F}(x))\right)\right)\right\| \\
& \leq\left\|P_{C_{k}}\left(x-\tau \mathscr{F}\left(P_{C_{k}}(x-\tau \mathscr{F}(x))\right)\right)-P_{C_{k}}\left(x-\tau \mathscr{F}\left(P_{C}(x-\tau \mathscr{F}(x))\right)\right)\right\| \\
& +\left\|P_{C_{k}}\left(x-\tau \mathscr{F}\left(P_{C}(x-\tau \mathscr{F}(x))\right)\right)-P_{C}\left(x-\tau \mathscr{F}\left(P_{C}(x-\tau \mathscr{F}(x))\right)\right)\right\| \\
& \leq\left\|-\tau \mathscr{F}\left(P_{C_{k}}(x-\tau \mathscr{F}(x))\right)+\tau \mathscr{F}\left(P_{C}(x-\tau \mathscr{F}(x))\right)\right\| \\
& +\left\|P_{C_{k}}\left(x-\tau \mathscr{F}\left(P_{C}(x-\tau \mathscr{F}(x))\right)\right)-P_{C}\left(x-\tau \mathscr{F}\left(P_{C}(x-\tau \mathscr{F}(x))\right)\right)\right\| .
\end{aligned}
$$

Since $\mathscr{F}$ is $\alpha$-ISM on $C$, it is Lipschitz continuous on $C$ with constant $1 / \alpha$. Therefore,

$$
\begin{aligned}
\left\|T_{k}(x)-T(x)\right\| & \leq \frac{\tau}{\alpha}\left\|P_{C}(x-\tau \mathscr{F}(x))-P_{C_{k}}(x-\tau \mathscr{F}(x))\right\| \\
& +\left\|P_{C_{k}}\left(x-\tau \mathscr{F}\left(P_{C}(x-\tau \mathscr{F}(x))\right)\right)-P_{C}\left(x-\tau \mathscr{F}\left(P_{C}(x-\tau \mathscr{F}(x))\right)\right)\right\| .
\end{aligned}
$$

Thus,

$$
D_{\gamma}\left(T_{k}, T\right) \leq \frac{\tau}{\alpha} d_{\bar{\gamma}}\left(C_{k}, C\right)+d_{\bar{\gamma}}\left(C_{k}, C\right)
$$

where $\bar{\gamma} \geq \max \left\{\|x-\tau \mathscr{F}(x)\|,\left\|x-\tau \mathscr{F}\left(P_{C}(x-\tau \mathscr{F}(x))\right)\right\|\right\}$. Since $C_{k} \stackrel{\text { epi }}{\rightarrow} C$, we have that $d_{\gamma}\left(C_{k}, C\right) \rightarrow 0$ for $\gamma \geq 0$ and the desired result is obtained. 
Remark 3.1. Observe that Proposition 3.1 holds with Lipschitz continuity on $C$ instead of $\alpha$-ISM.

We now establish convergence of Algorithm 3.2.

Theorem 3.2. Let $C$ and $\left\{C_{k}\right\}_{k=0}^{\infty}$ be a set and a sequence of sets in $\operatorname{NCCS}\left(\mathbb{R}^{n}\right)$ such that $C_{k} \subseteq C_{k+1} \subseteq C$ and $C_{k} \stackrel{\text { epi }}{\rightarrow}$ C. Assume that Conditions Conditions 3.1-3.3 hold and choose $\tau \in(0,1 / \alpha)$. In addition, assume that for any $\gamma \geq 0$

$$
\sum_{k=0}^{\infty} \alpha_{k}\left(\frac{\tau}{\alpha} d_{\gamma}\left(C_{k}, C\right)+d_{\gamma}\left(C_{k}, C\right)\right)<+\infty
$$

and that the $\alpha_{k} \in(0,1)$ satisfy $\sum_{k=0}^{\infty} \alpha_{k}\left(1-\alpha_{k}\right)=+\infty$. Then any sequence $\left\{x^{k}\right\}_{k=0}^{\infty}$ generated by Algorithm 3.2 converges to a point $x^{*} \in \operatorname{Sol}(\mathscr{F}, C)$.

Proof. By Propositions 3.1 and 3.2, $T$ and $\left\{T_{k}\right\}_{k=0}^{\infty}$ are non-expansive mappings and $T_{k} \rightarrow T$. Applying the assumption (3.12) to (3.11) we get $\sum_{k=0}^{\infty} \alpha_{k} D_{\gamma}\left(T_{k}, T\right)<+\infty$ and therefore, all the conditions of Theorem 2.4 are fulfilled. Since $\operatorname{Sol}(\mathscr{F}, C) \neq \emptyset$ (Condition 3.1) and by (2.15) $\operatorname{Sol}(\mathscr{F}, C)=$ Fix $\left(P_{C}\left(I-\lambda \mathscr{F}\left(P_{C}(I-\lambda \mathscr{F})\right)\right)\right)=F i x(T)$ the desired result is obtained.

\section{SECOND RELAXED PERTURbED EXTRAGRADIENT ALGORITHM}

In this section we wish to propose another relaxed perturbed extragradient algorithm by defining

$$
\begin{aligned}
T_{k}(x) & :=P_{C_{k}}\left(P_{C_{k}}(x-\tau \mathscr{F}(x))-\tau \mathscr{F}\left(P_{C_{k}}(x-\tau \mathscr{F}(x))\right)\right), \\
& =\left[P_{C_{k}}(I-\tau \mathscr{F})\right]^{2}(x) .
\end{aligned}
$$

for in Algorithm 3.2. Observe that such idea for $T_{k}$ is not new and is presented by Noor in $[18,19]$ with $\mathscr{F}$ being monotone and Lipschitz continuities, but it can be proved that these assumptions does not guarantee convergence and moreover

$$
\operatorname{Sol}(\mathscr{F}, C) \neq \operatorname{Fix}\left(\left[P_{C}(I-\tau \mathscr{F})\right]^{2}\right) .
$$

The next example in this matter is proposed to us by Professor Charlie Byrne.

Example 4.1. Let $\mathscr{F}$ be the operator on $\mathbb{R}^{2}$ given by multiplication by the matrix

$$
\mathscr{F}=\left[\begin{array}{cc}
0 & a \\
-a & 0
\end{array}\right],
$$

for some $a \in(0,1)$. The operator $\mathscr{F}$ is then monotone and $a$-Lipschitz continuous. With $C=\mathbb{R}^{2}$, the variational inequality problem is then equivalent to finding a zero of $\mathscr{F}$. Note that $\mathscr{F}(z)=0$ if and only if $z=0$.

The Korpelevich iteration in this case (with $\tau=1$ ) is

$$
x^{k+1}=T\left(x^{k}\right)=(I-\mathscr{F}(I-\mathscr{F})) x^{k} .
$$

On the other hand (Noor in $[18,19])$ we have the iterative step:

$$
x^{k+1}=P\left(x^{k}\right)=(I-\mathscr{F})^{2} x^{k} .
$$

The operator $T$ is then multiplication by the matrix

$$
T=\left[\begin{array}{cc}
1-a^{2} & -a \\
a & 1-a^{2}
\end{array}\right],
$$


and the operator $P$ is multiplication by the matrix

$$
P=\left[\begin{array}{cc}
1-a^{2} & -2 a \\
2 a & 1-a^{2}
\end{array}\right]
$$

For any $x \in \mathbb{R}^{2}$ we have

$$
\|T(x)\|^{2}=\left(\left(1-a^{2}\right)^{2}+a^{2}\right)\|x\|^{2}<\|x\|^{2},
$$

for all $x \neq 0$, while

$$
\|P(x)\|^{2}=\left(\left(1-a^{2}\right)^{2}+4 a^{2}\right)\|x\|^{2}=\left(1+a^{2}\right)^{2}\|x\|^{2} .
$$

This proves that the sequence $x^{k+1}=P\left(x^{k}\right)$ does not converge, generally.

We show how by assuming inverse-strongly monomnoicity is needed for this variant. The full convergence theorem is given next.

Lemma 4.1. Let $C \subset \mathbb{R}^{n}$ be non-empty, closed and convex. Let $\mathscr{F}: \mathbb{R}^{n} \rightarrow \mathbb{R}^{n}$ be $\alpha$-ISM, then for any $\tau \in(0,2 \alpha)$, we get

$$
\operatorname{Sol}(\mathscr{F}, C)=F i x\left(\left[P_{C}(I-\tau \mathscr{F})\right]^{2}\right) .
$$

Proof. (i) Let $x \in \operatorname{Sol}(\mathscr{F}, C)$. Applying (2.8) twice, we get

$$
P_{C}\left(P_{C}(x-\tau \mathscr{F}(x))-\tau \mathscr{F}\left(P_{C}(x-\tau \mathscr{F}(x))\right)\right)=P_{C}(I-\tau \mathscr{F}(x))=x
$$

which implies that $x \in$ Fix $\left(\left[P_{C}(I-\tau \mathscr{F})\right]^{2}\right)$.

(ii) On the other hand, let $x \in F i x\left(\left[P_{C}(I-\tau \mathscr{F})\right]^{2}\right)$. Denote by $y:=P_{C}(x-\tau \mathscr{F}(x))$, we get $x=P_{C}(y-$ $\tau \mathscr{F}(y))$. We now show that $x=y$. Indeed, following the non-expansiveness of the metric projection $P_{C}$ and the $\alpha$-ISM of $\mathscr{F}$

$$
\begin{aligned}
\|x-y\|^{2} & =\left\|P_{C}(y-\tau \mathscr{F}(y))-P_{C}(x-\tau \mathscr{F}(x))\right\|^{2} \\
& \leq\|(y-\tau \mathscr{F}(y))-(x-\tau \mathscr{F}(x))\| \\
& =\|x-y\|^{2}+\tau^{2}\|\mathscr{F}(x)-\mathscr{F}(y)\|^{2} \\
& -2 \tau\langle x-y, \mathscr{F}(x)-\mathscr{F}(y)\rangle \\
& \leq\|x-y\|^{2}+\tau(\tau-2 \alpha)\|\mathscr{F}(x)-\mathscr{F}(y)\|^{2} \\
& \leq\|x-y\|^{2}
\end{aligned}
$$

following the assumption on $\tau$ we get that $x=y$, meaning that $x=y=P_{C}(x-\tau \mathscr{F}(x))$, i.e., $x \in \operatorname{Sol}(\mathscr{F}, C)$.

Now the non-expansiveness of $T$ and $T_{k}$ is trivial as a power of the non-expansive operators $P_{C}(I-$ $\tau \mathscr{F})$ and $P_{C_{k}}(I-\tau \mathscr{F})$. Here again the non-expansiveness of $P_{C}(I-\tau \mathscr{F})$ and $P_{C_{k}}(I-\tau \mathscr{F})$ requires that $\mathscr{F}$ is $\alpha$-ISM!

Proposition 4.1. Assume that $C_{k} \subseteq C_{k+1} \subseteq C$ such that $C_{k} \stackrel{\text { epi }}{\rightarrow} C$. In addition, assume that $\mathscr{F}$ is $\alpha$-ISM on $C$ and choose $\tau \in(0,2 \alpha)$. Then $T_{k} \rightarrow T$. 
Proof. Let $x \in \mathbb{R}^{n}$ and let $\|x\| \leq \gamma$ for $\gamma \geq 0$. Denote $z:=x-\tau \mathscr{F}(x)$. Then, following the nonexpansiveness of the metric projections $P_{C}$ and $P_{C_{k}}$ we obtain.

$$
\begin{aligned}
\left\|T_{k}(x)-T(x)\right\| & =\left\|P_{C_{k}}\left(P_{C_{k}}(z)-\tau \mathscr{F}\left(P_{C_{k}}(z)\right)\right)-P_{C}\left(P_{C}(z)-\tau \mathscr{F}\left(P_{C}(z)\right)\right)\right\| \\
& \leq\left\|P_{C_{k}}\left(P_{C_{k}}(z)-\tau \mathscr{F}\left(P_{C_{k}}(z)\right)\right)-P_{C_{k}}\left(P_{C}(z)-\tau \mathscr{F}\left(P_{C}(z)\right)\right)\right\| \\
& +\left\|P_{C_{k}}\left(P_{C}(z)-\tau \mathscr{F}\left(P_{C}(z)\right)\right)-P_{C}\left(P_{C}(z)-\tau \mathscr{F}\left(P_{C}(z)\right)\right)\right\| \\
& \leq\left\|\left(P_{C_{k}}(z)-\tau \mathscr{F}\left(P_{C_{k}}(z)\right)\right)-\left(P_{C}(z)-\tau \mathscr{F}\left(P_{C}(z)\right)\right)\right\| \\
& +\left\|P_{C_{k}}\left(P_{C}(z)-\tau \mathscr{F}\left(P_{C}(z)\right)\right)-P_{C}\left(P_{C}(z)-\tau \mathscr{F}\left(P_{C}(z)\right)\right)\right\| \\
& \leq\left\|P_{C_{k}}(z)-P_{C}(z)\right\|+\left\|\tau\left(\mathscr{F}\left(P_{C}(z)\right)-\mathscr{F}\left(P_{C_{k}}(z)\right)\right)\right\| \\
& +\left\|P_{C_{k}}\left(P_{C}(z)-\tau \mathscr{F}\left(P_{C}(z)\right)\right)-P_{C}\left(P_{C}(z)-\tau \mathscr{F}\left(P_{C}(z)\right)\right)\right\| \\
& \leq\left\|P_{C_{k}}(z)-P_{C}(z)\right\|+\frac{\tau}{\alpha}\left\|P_{C}(z)-P_{C_{k}}(z)\right\| \\
& +\left\|P_{C_{k}}\left(P_{C}(z)-\tau \mathscr{F}\left(P_{C}(z)\right)\right)-P_{C}\left(P_{C}(z)-\tau \mathscr{F}\left(P_{C}(z)\right)\right)\right\| \\
& =\left(1+\frac{\tau}{\alpha}\right)\left\|P_{C}(x-\tau \mathscr{F}(x))-P_{C_{k}}(x-\tau \mathscr{F}(x))\right\| \\
& +\left\|P_{C_{k}}\left(P_{C}(z)-\tau \mathscr{F}\left(P_{C}(z)\right)\right)-P_{C}\left(P_{C}(z)-\tau \mathscr{F}\left(P_{C}(z)\right)\right)\right\| .
\end{aligned}
$$

Therefore,

$$
\begin{aligned}
\left\|T_{k}(x)-T(x)\right\| & \leq\left(1+\frac{\tau}{\alpha}\right)\left\|P_{C}(x-\tau \mathscr{F}(x))-P_{C_{k}}(x-\tau \mathscr{F}(x))\right\| \\
& +\left\|\begin{array}{c}
P_{C_{k}}\left(P_{C}(x-\tau \mathscr{F}(x))-\tau \mathscr{F}\left(P_{C}(x-\tau \mathscr{F}(x))\right)\right) \\
-P_{C}\left(P_{C}(x-\tau \mathscr{F}(x))-\tau \mathscr{F}\left(P_{C}(x-\tau \mathscr{F}(x))\right)\right)
\end{array}\right\| .
\end{aligned}
$$

Thus,

$$
D_{\gamma}\left(T_{k}, T\right) \leq\left(1+\frac{\tau}{\alpha}\right) d_{\bar{\gamma}}\left(S_{k}, S\right)+d_{\bar{\gamma}}\left(S_{k}, S\right),
$$

where $\bar{\gamma} \geq \max \left\{\|x-\tau \mathscr{F}(x)\|,\left\|P_{C}(x-\tau \mathscr{F}(x))-\tau \mathscr{F}\left(P_{C}(x-\tau \mathscr{F}(x))\right)\right\|\right\}$. Since $S_{k} \stackrel{\text { epi }}{\rightarrow} S$, we have that $d_{\gamma}\left(S_{k}, S\right) \rightarrow 0$ for $\gamma \geq 0$ and the desired result is obtained.

Theorem 4.1. Let $C$ and $\left\{C_{k}\right\}_{k=0}^{\infty}$ be a set and a sequence of sets in $N C C S\left(\mathbb{R}^{n}\right)$ such that $C_{k} \subseteq C_{k+1} \subseteq C$ and $C_{k} \stackrel{e p i}{\rightarrow} C$. Assume that $\operatorname{Sol}(\mathscr{F}, C) \neq \emptyset$, that $\mathscr{F}$ is $\alpha-I S M$ on $C$ and choose $\tau \in(0,2 \alpha)$. In addition, assume that for any $\gamma \geq 0$

$$
\sum_{k=0}^{\infty} \alpha_{k}\left(\left(1+\frac{\tau}{\alpha}\right) d_{\gamma}\left(C_{k}, C\right)+d_{\gamma}\left(C_{k}, S\right)\right)<+\infty
$$

and that the $\alpha_{k} \in(0,1)$ satisfy $\sum_{k=0}^{\infty} \alpha_{k}\left(1-\alpha_{k}\right)=+\infty$. Then any sequence $\left\{x^{k}\right\}_{k=0}^{\infty}$ generated by Algorithm 3.2 converges to a point $x^{*} \in \operatorname{Sol}(\mathscr{F}, C)$.

\section{Acknowledgements}

The author would like to thank Prof. Charlie Byrne for his time and effort corresponding on this paper, his useful comments, examples and suggestions helped in the preparation of this work. This work was supported by the EU FP7 IRSES program STREVCOMS, grant no. PIRSES-GA-2013-612669. 


\section{REFERENCES}

[1] A.S. Antipin, On a method for convex programs using a symmetrical modification of the Lagrange function, Ekonomika i Mat. Metody 12 (1976), 1164-1173.

[2] H. Attouch, Variational Convergence for Functions and Operators, Pitman, London, 1984.

[3] H. Attouch, R. B. Wets, Isometries for the Legendre-Fenchel transform, Trans. Amer. Math. Soc. 296 (1986), 33-60.

[4] J.-B. Baillon, R. E. Bruck, S. Reich, On the asymptotic behavior of non-expansive mappings and semigroups in Banach spaces, Houston J. Math. 4 (1978), 1-9.

[5] J. M. Borwein, A. S. Lewis, Convex Analysis and Nonlinear Optimization Theory and Examples, Springer-Verlag, NewYork, 2000.

[6] C. L. Byrne, A unified treatment of some iterative algorithms in signal processing and image reconstruction, Inverse Probl. 20 (2004), 103-120.

[7] A. Cegielski, A generalization of the Opial's Theorem, Control Cybern. 36 (2007), 601-610.

[8] A. Cegielski, Y. Censor, Opial-type theorems and the common fixed point problem, in: H. H. Bauschke, R. S. Burachik, P.L. Combettes, V. Elser, D. R. Luke and H. Wolkowicz (Editors), Fixed-Point Algorithms for Inverse Problems in Science and Engineering, Springer, New York, 155-183, 2011.

[9] A. Cegielski, Iterative Methods for Fixed Point Problems in Hilbert Spaces, Lecture Notes in Mathematics 2057, Springer, Heidelberg, 2012.

[10] Y. Censor, A. Gibali, S. Reich, Extensions of Korpelevich's extragradient method for solving the variational inequality problem in Euclidean space, Optimization 61 (2012), 1119-1132.

[11] B. C. Eaves, On the basic theorem of complementarity, Mat. Program. 1 (1971), 68-75.

[12] F. Facchinei, J. S. Pang, Finite-Dimensional Variational Inequalities and Complementarity Problems, Volume I and Volume II, Springer-Verlag, New York, NY, USA, 2003.

[13] K. Goebel, S. Reich, Uniform Convexity, Hyperbolic Geometry, and Nonexpansive Mappings, Marcel Dekker, New York and Basel, 1984.

[14] M. M. Israel Jr., S. Reich, Extension and selection problems for nonlinear semigroups in Banach Spaces, Math. Japon. 28 (1983), 1-8.

[15] G. M. Korpelevich, The extragradient method for finding saddle points and other problems, Ekonomika i Matematcheskie Metody 12 (1976), 747-756.

[16] M. A. Krasnosel'skiǔ, Two remarks on the method of successive approximations, (in Russian), Uspekhi Matematicheskikh Nauk 10 (1955), 123-127.

[17] W. R. Mann, Mean value methods in iteration, Proc. Amer. Math. Soc. 4 (1953), 506-510.

[18] M. A. Noor, Some algorithms for general monotone mixed variational inequalities, Math. Comuput. Model. 29 (1999), $1-9$.

[19] M. A. Noor, Some development in general variational inequalities, Appl. Math. Comput. 152 (2004), 199-277.

[20] Z. Opial, Weak convergence of the sequence of successive approximations for nonexpansive mappings, Bull. Amer. Math. Soc. 73 (1967), 591-597.

[21] P. S. M. Santos, S. Scheimberg, A projection algorithm for general variational inequalities with perturbed constraint set, Appl. Math. Comput. 181 (2006), 649-661.

[22] A. J. Zaslavski, Numerical Optimization with Computational Errors, Springer, 2016.

[23] Q. Yang, J. Zhao, Generalized KM theorems and their applications, Inverse Probl. 22 (2006), 833-844. 